\title{
Errata
}

\section{Correction to "Comparison and Validation of Tissue Modelization and Statistical Classification Methods in T1-Weighted MR Brain Images"}

In [1], the web addresses for O. Cuisenaire and J.-P. Thiran were misidentified. Theses should have read as http://itswww.epfl.ch and http://lts5www.epfl.ch, respectively.

In Section III-A, the initialization step should have read as follows. Initialization Step: Choose the best initialization for $\hat{\theta}^{0}$.

Section VI-A (Global Performance) should have read as follows.

There is no global winner as the most suitable tissue classification technique for T1-MR brain image. In fact, if we define the best classification as the one with the highest percentage of correct classified voxels, as in Fig. 7, the optimal method varies depending on the noise (N) and in-homogeneity (RF) levels present in the images. For low noise levels $(N \leq 3 \%)$, no method clearly outperforms the others. However, for higher noise levels ( $N \geq 5 \%$ ), D-GPV-HMRF almost always performs the best classification, closely followed by B-GHMRF, whose performance differs by less than $2 \%$. In [46], methods are also compared by allowing small errors such as confusing a pure tissue with a PV containing it or confusing a PV voxel with one of its pure tissues. In this case, C-GPV and D-GPV-HMRF, both methods using the PV equation, have the lowest error rates for low and high noise levels, respectively. However, differences are less than $1 \%$.

The final paragraph of Section VI-B (Robustness to Noise and Inhomogeneities) should have read as follows.

Solid lines represent all methods using local spatial priors, which present similar behaviors with noise and bias. With no bias field, $\mathrm{RF}=$ 0 , pergood decreases proportionally to the increase of noise. For $\mathrm{RF}=$ 20 , there is no decrease of quality but almost a constant pergood. Finally, for $\mathrm{RF}=40$, the pergood actually increases for high noise levels. The reason for this unexpected behavior is that-in the presence of a strong bias field- low noise levels ( $\mathrm{N} \leq 3 \%$ ) are not realistically modeled by Gaussian distributions.

Manuscript received December 8, 2005.

Digital Object Identifier 10.1109/TMI.2006.869963
The first paragraph of Section VI-C (Pure Tissues and Partial Volume) should have read as follows.

Using confusion tables such as Table III, the global conclusions can be refined on a tissue per tissue basis. Considering such confusion tables for noises $N \geq 5 \%$ and bias fields $\mathrm{RF}=0 \%, \mathrm{RF}=20 \%$, and $\mathrm{RF}=40 \%$, we observe that the best classifier for CSF is B-GHMRF (70\% of the cases), the best classifier for GM is F-NP-HMRF (70\% of the cases) and the best classification of WM tissue is performed by B-GHMRF in more than 50\% of the cases. D-GPV-HMRF almost always achieves the best classification score for both PV tissues: $78 \%$ of the cases for CG and $100 \%$ for GW.

\section{REFERENCES}

[1] M. Bach Cuadra, L. Cammoun, T. Butz, O. Cuisenaire, and J.-P. Thiran, "Comparison and validation of tissue modelization and statistical classification methods in T1-weighted MR brain images," IEEE Trans. Med. Imag., vol. 25, no. 12, pp. 1548-1565, Dec. 2005.

\section{Correction to "Biventricular Myocardial Strains via Nonrigid Registration of Anatomical NURBS Models"}

The title of [1] became corrupt during production. The title should have read Biventricular Myocardial Strains via Nonrigid Registration of Anatomical NURBS Model.

\section{REFERENCES}

[1] N. J. Tustison and A. A. Amini, "Biventricular myocardial strains via nonrigid registration of anatomical NURBS models," IEEE Trans. Med. Imag., vol. 25, no. 1, pp. 94-112, Jan. 2006.

Manuscript received December 30, 2005.

Digital Object Identifier 10.1109/TMI.2006.870010 